\title{
Increasing the effectiveness of electrical resistivity tomography using $\gamma_{11 \mathrm{n}}$ configurations
}

\author{
S. Szalai*, Á. Kis, M. Metwaly, I. Lemperger and K. Szokoli \\ RCAES HAS, GGI, H-9401 Sopron POB 5, Hungary
}

Received October 2013, revision accepted September 2014

\begin{abstract}
A new array type, i.e., the $\gamma_{11 \mathrm{n}}$ arrays, is introduced in this paper, in which the sequence of the current $(\mathrm{C})$ and potential $(\mathrm{P})$ electrodes is $\mathrm{CPCP}$, and the distance between the last two electrodes is $n$ times the distance between the first two ones and that of the second one and the third one. These arrays are called quasinull arrays because they are-according to their array and behaviour-between the traditional and null arrays. It is shown by numerical modelling that, in detecting small-effect inhomogeneity, these configurations may be more effective than the traditional ones, including the optimized Stummer configuration. Certain $\gamma_{11 \text { n }}$ configurations-especially the $\gamma_{112}, \gamma_{113}$, and $\gamma_{114}$-produced better results both in horizontal and vertical resolution investigations. Based on the numerical studies, the $\gamma_{11 \mathrm{n}}$ configurations seem to be very promising in problems where the anomalies are similar to the numerically investigated ones, i.e., they can detect and characterize, e.g., tunnels, caves, cables, tubes, abandoned riverbeds, or discontinuity, in a clay layer with greater efficacy than those of the traditional configurations. $\gamma_{11 \mathrm{n}}$ measurements need less data than traditional configurations; therefore, the time demand of electrical resistivity tomography measurements can be shortened by their use.
\end{abstract}

Key words: Geoelectric configuration, $\gamma_{11 n}$ configurations, Depth of detectability, ERT, Quasinull arrays.

\section{INTRODUCTION}

Geoelectric methods form a traditional group of geophysical techniques (Van Nostrand and Cook 1966; Alpin et al. 1966; Zhdanov and Keller 1993). In the early times, their use was restricted to mineral exploration. Today they are frequently used in numerous field problems (Butler 2005), which are related to electrical resistivity distribution of the subsurface: hydrogeology (Kirsch 2006), environmental studies (Ward 1990; Knödel, Krummel, and Lange 2005), engineering (Ward 1990, Szalai et al., 2009a), safety purposes (Metwaly et al. 2008), archaeological problems (Clark 1990), etc,

The number of published geoelectric arrays used for geoelec-tric measurements is more than 100 (Szalai and Szarka

*E-mail: szalai@ggki.hu 2008a). It is widely known (mainly from Ward 1990) that each array has some specific advantages and disadvantages. In studying these qualities, the arrays were compared from many different aspects. One of the key parameters, i.e., the depth of the investigation, value was calculated by Szalai et al. (2009b) following the slight-ly different definitions given by Edwards (1977) and Roy and Apparao (1971) for all arrays. Parameter sensitivity maps, which are crucial in understanding the different arrays, were presented by Szalai and Szarka (2008b,2008c) for all arrays that ever existed. Ward (1990) evalu-ated the geoelectric arrays from 14 various aspects. Although the aforementioned investigations aimed at providing a theoretical basis for traditional profiling and sounding techniques, they are also important for electrical resistivity tomography (ERT) meas-urements because the individual arrays serve as a basis for the ERT measurements. 
Since ERT measurements have become the dominant tool in geoelectric research in the past decades, it is of crucial impor-tance to maximize the information available when using them. There are actually significant efforts to find the best possible, so-called optimized configurations (Furman Furman A., Ferré, and Warrick 2003; Stummer, Maurer and Green 2004; Wilkinson et al. 2006). The optimized configurations, e.g., the Stummer configuration (Stummer et al. 2004) - in contrast to the classical configuration approach-may contain a series of very different arrays.

Stummer et al. (2004) did not however include in the optimization procedure $\gamma$-type arrays and therefore not the $\gamma_{11 \mathrm{n}}$ arrays. In the case of these arrays, the electrodes are positioned in an overlapping mode that is the current, and potential electrodes follow each other alternately (see Fig. 1). The large value of the $k$ geometrical factor does not, however, inevitably refer to the field applicability of an array as shown by Szalai et al. (2002) and Szalai et al. (2004). $k$ is namely the function of the homogeneous half-space value that has nothing to do with the potential due to the inhomogeneity that contains information that is important for us. If the $\gamma_{11 \mathrm{n}}$ arrays will prove to be useful, they have to be taken into account in all optimization processes.

In the last few years, several other motivations were accumulated to study the $\gamma_{11 \mathrm{n}}$ and $\gamma \mathrm{m}_{11 \mathrm{n}}$ configurations, which will be discussed in the next section. First, the definition of the applied non-conventional arrays is given. The $\gamma_{11 \mathrm{n}}$ arrays are presented in Fig. 1. A $\gamma \mathrm{m}_{11 \mathrm{n}}$ (mirrored $\gamma_{11 \mathrm{n}}$ ) array contains a $\gamma_{11 \mathrm{n}}$ array and its pair, a $\gamma_{\mathrm{n} 11}$ array. These arrays are the same, but they are orientated in opposite directions carrying out measurements. The $\gamma \mathrm{m}_{11 \mathrm{n}}$ configuration that consists of $\gamma \mathrm{m}_{11 \mathrm{n}}$ arrays creates a data set that is twice more dense than the $\gamma_{11 \mathrm{n}}$ configuration in itself. $\gamma(\mathrm{m})_{11 \mathrm{n}}$ refers to both the $\gamma_{11 \mathrm{n}}$ and $\gamma \mathrm{m}_{11 \mathrm{n}}$ configurations.

\section{MOTIVATIONS TO STUDY THE $\gamma_{11 \mathrm{~N}}$ AND $\gamma \mathrm{m}_{11 \mathrm{~N}}$ CONFIGURATIONS}

(i) Furman et al. (2003) performed a sensitivity analysis and demonstrated the supremacy of the "partially overlapping arrays," which are also $\gamma$-type arrays, i.e., their electrode sequence is CPCP. Szalai and Szarka (2008b) presented the normalized parameter sensitivity (nPS) maps of many linear arrays. Many of them are reproduced in Fig. 2. In the first row, the nPS maps of the Wenner- $\alpha$ (W- $\alpha$ ), Wenner- $\beta$ $(\mathrm{W}-\beta)$, dipole-axial (Dp-ax) four-electrode, and the $\mathrm{P}-\mathrm{Dp}$ three-electrode arrays are shown in a depth of one tenth of the array length. (The array length is the distance of the farthest electrodes that are not in the infinity.) In the second row, the
nPS maps of several characteristic $\gamma_{\mathrm{n} 11}$ arrays are presented, which are the oppositely orientated versions of the $\gamma_{11 \mathrm{n}}$ arrays; therefore, their nPS map is also the oppositely orientated version of those of the $\gamma_{11 \mathrm{n}}$ arrays. The first one, i.e., the $\gamma_{111}$ array, (which is $n=1$ ) is in fact the Wenner- $\gamma$ array, i.e., a traditional array. The last one, i.e., the $\gamma_{\mathrm{n} 11}$ array, where $n$ $=\infty$, is a null array, i.e., the midpoint-null or MAN array (Szalai et al. 2004). The $\gamma_{811}$ array is one of the series of the $\gamma_{\mathrm{n} 11}$ arrays between the traditional and null arrays. Similar arrays $(n=1-7)$, which have similar nPS maps, are investigated in this paper. Its maximal value is shown, below each nPS map. It is well seen that, although the $\gamma_{111}$ array's value is in the same order than those of the values of the first row arrays, the maximal values of the $\gamma_{\mathrm{n} 11}$ nPS maps are drastically increasing with increasing $n$. This high sensitivity motivated us to study the depth of detectability (DD) value of these arrays. (ii) The calculations of the DD values by Szalai et al. (2013b) has shown that the DD values of the $\gamma_{11 n}$ configurations can be 2-2.5 times larger than that of the best traditional configuration. A square resistive prism (e.g., $2 \times 2 \mathrm{~m}$ cross-section, $200-\Omega \mathrm{m}$ resistivity in a host of $100 \Omega \mathrm{m}$ ) proved to be detectable from a depth of $14 \mathrm{~m}$ (upper side of the prism), assuming 5\% noise level and using $\gamma_{113}$ configuration, whereas it was detectable by the best traditional configuration, the P-Dp one, only from a depth of $6.6 \mathrm{~m}$. In the investigation, 100 electrodes were applied with an electrode distance of $1 \mathrm{~m}$. By applying these configurations, one could therefore get information from a larger depth that can be especially important in areas where the space available for measurements is limited, e.g., in built-in areas.

(iii) It was shown by comparing Szalai et al. (2011) and Szalai et al. (2013a) that, the higher the DD value of a configuration, the better are its imaging features. Among the traditional configurations, the $\mathrm{DD}$ value of the $\beta$-type configurations ( $\mathrm{Dp}-$ $\mathrm{Dp}, \mathrm{P}-\mathrm{Dp}$, Stummer, Wenner- $\beta$, electrode sequence is CCPP) was generally larger than those of the other configurations (Szalai et al. 2011). According to the imaging capacity, the sequence of the investigated configurations was: Stummer, Dp-Dp, Wenner- $\beta$, P-Dp, Wenner- $\alpha$, and P-P (Szalai et al. 2013a). That is the ( $\beta$-type) configurations that have larger DD values proved to have better imaging capacity.

Concluding from (i), (ii), and (iii), one can say that the larger $\mathrm{nPS}_{\max }$ value may lead to larger DD values, which may result in better imaging capacity. The high $\mathrm{nPS}_{\max }$ values of the $\gamma_{11 \mathrm{n}}$ configurations could therefore result in good imaging features of these configurations.

(iv) Szalai et al. (2002) and Falco et al. (2013) demonstrated that geometrical null arrays (null arrays that provide zero 


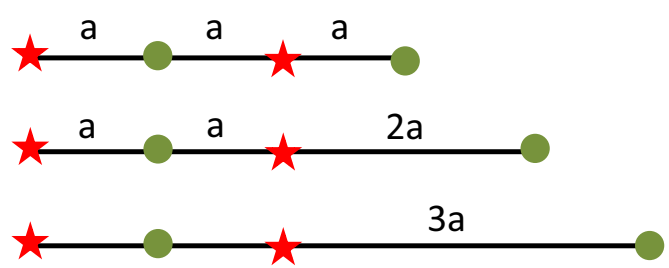

$\gamma_{111}$

$\gamma_{112}$

$\gamma_{113}$
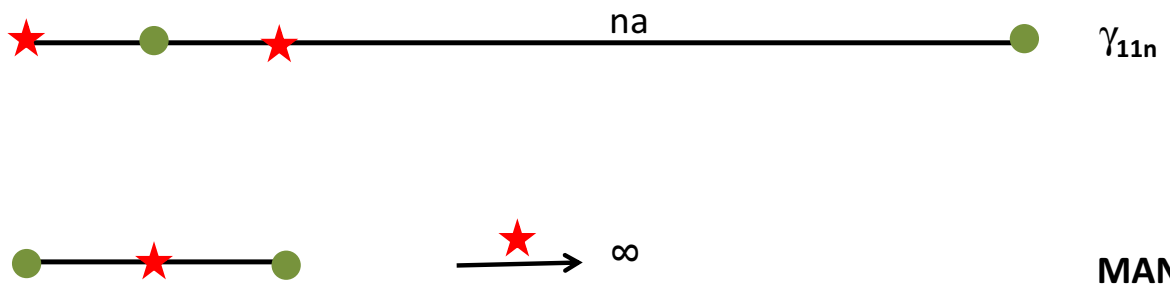

$M A N=\gamma_{11 n}$

$n=$ inf

Figure 1 The $\gamma_{11 \mathrm{n}}$ arrays. Stars denote current, circles denote potential electrodes, and $a$ is the electrode distance.

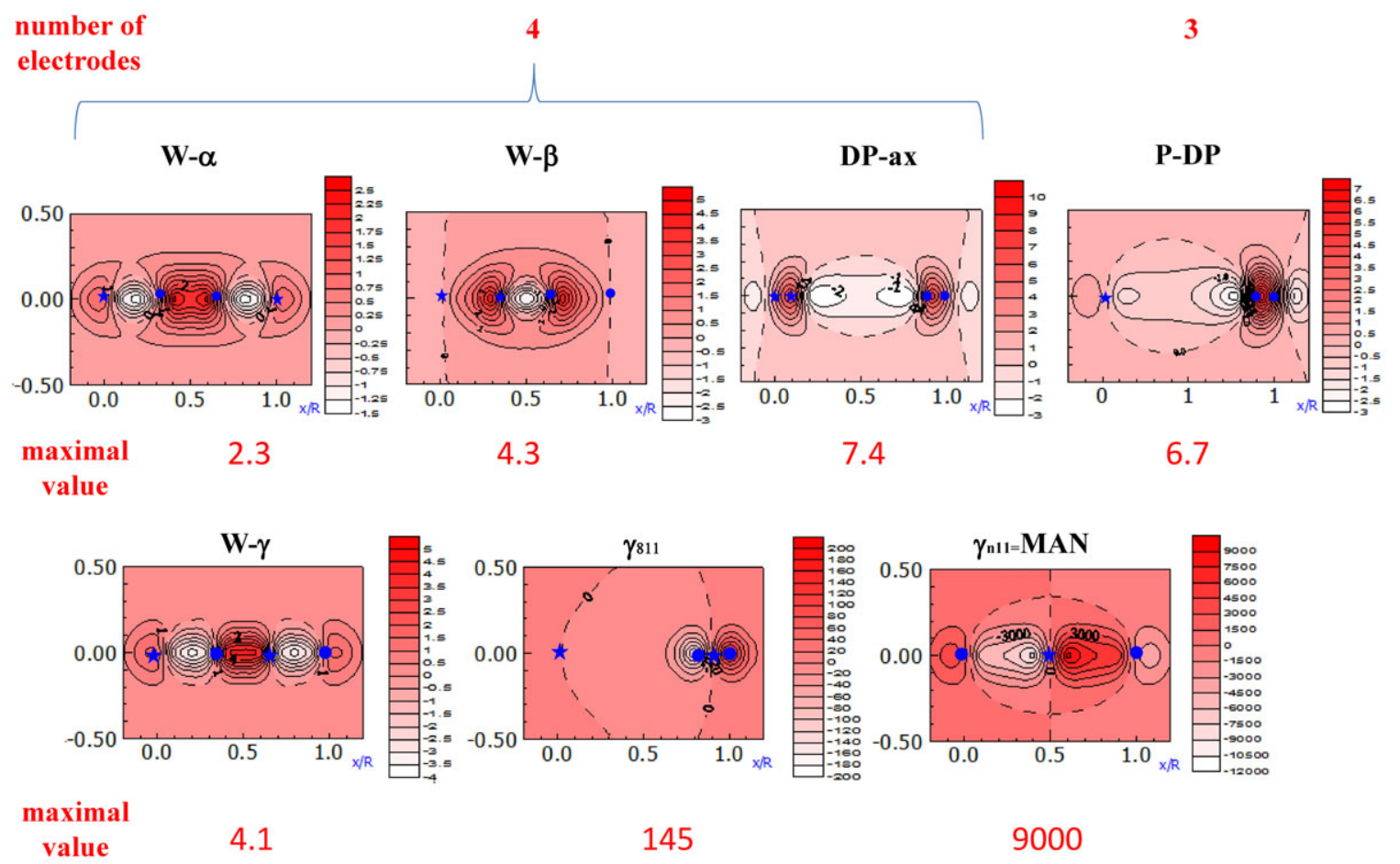

Figure 2 The nPS maps of several traditional and typical $\gamma_{\mathrm{n} 11}$ arrays in a depth of one tenth of the array length. Stars denote current, and circles denote potential electrodes. Below the maps, their maximal values can be seen.

signal in homogeneous half-space due to the appropriate positioning of the electrodes) can be very effective in field conditions. There is only one geometrical null array that can be built in 2D multi-electrode systems, the MAN array (Szalai et al. 2004 and Fig. 1). The applicability of the commercial software to invert its data is very limited yet or not possible, whereas $\gamma_{11 n}$ configuration data may be inverted among certain conditions. Due to the fact that the MAN array is a 
special case of this array type if $n$ is infinite (see Fig. 1), the investigation of $\gamma_{11 \mathrm{n}}$ arrays can be also very useful to progress in understanding better the MAN array. According to numerical calculations by Szalai et al. (2004), even the signal strength of the $\gamma_{11 \mathrm{n}}$ arrays may be larger than that of the traditional arrays. About a dyke for example, with increasing depth, the size of the anomaly was $50 \Omega \mathrm{m}, 20 \Omega \mathrm{m}$, and $12 \Omega \mathrm{m}$ with the most appropriate array lengths for the Wenner- $\alpha$ array, whereas it was about $60 \Omega \mathrm{m}, 36 \Omega \mathrm{m}$, and $32 \Omega \mathrm{m}$ for the MAN array, accordingly. It can be clearly seen that, with the increasing depth of the dyke, the MAN array's signal strength became even better and better in comparison with that of the traditional array. Even in field situation, in Finland, although the traditional Wenner- $\alpha$ array had larger signal strength, both the MAN (it was called Midpoint-null array) and the Wenner- $\gamma$ null arrays produced larger anomalies due to horizontal resistivity changes (Szalai et al. 2004).

Based on these experiences, the study of the $\gamma(\mathrm{m})_{11 \mathrm{n}}$ configurations seems to be very reasonable.

To understand these arrays, it is very important to see that they used to produce very sharp anomalies as can be expected based on Fig. 2 and as it was verified in both numerical investigations and field measurements (Szalai et al. 2004). It means that their anomalies are very sensitive to the horizontal resistivity changes. Aside from 1D investigations, where the resistivity values supposed to change only in vertical direction, this is one of the most important factors of the imaging.

Unfortunately, the commercial inversion software tools are not able to maintain such sharp anomalies; therefore, the information that is contained in the measured data cannot be obtained. This is why, in this paper, mostly inhomogeneity with a small impact on the surface potential distribution is studied. In this way, the gradient of the signal will be not as large and, therefore, will be more easily followed by the inversion. The other reason to investigate such inhomogeneity is that the traditional arrays produce acceptable results for largeimpact inhomogeneity; therefore, for such problems, the application of other configurations is not required. In the future, a coarse image could be first obtained by a traditional configuration that could later serve as a priori model for $\gamma(\mathrm{m})_{11 \mathrm{n}}$ configurations to refine the inverted images.

\section{NUMERICAL INVESTIGATIONS}

\section{The investigated configurations}

The results obtained by the $\gamma_{11 n}$ (Fig. 1) and their mirrored version, i.e., the $\gamma \mathrm{m}_{11 \mathrm{n}}$ configurations, were compared in the paper with the results of traditionally used configurations such as the dipole-dipole (Dp-Dp), pole-dipole (P-Dp), Wenner- $\alpha$, and the optimized Stummer ones (Fig. 3).

In the investigation, configurations with 60 electrodes were used, with 1-m electrode spacing excluding the Stummer configuration. Because the Stummer configuration is available only for 30 electrodes (Stummer et al. 2004), an electrode distance of $2 \mathrm{~m}$ was used to get the same configuration length as for the other configurations. The number of its data points is even in this case greater than that of any $\gamma(\mathrm{m})_{11 \mathrm{n}}$ configurations, excluding only the $\gamma \mathrm{m}_{112}$ one (see Figs. 3 and 4 ). It has also to be noted that, in spite of the same change, the Stummer configuration proved to be the best traditional configuration in the investigations by Szalai et al. (2013). It is also possible that the imaging quality of the Stummer configuration could be further improved by using the same electrode distance as for the other configurations, but it would lead to a significant increase in the data number. Moreover, the imaging quality of the $\gamma(\mathrm{m})_{11 \mathrm{n}}$ configurations could most likely be improved by combining them.

The Dp-Dp configuration was used because it is the best traditional configuration in the investigations, as proved by Szalai et al. (2013a). The P-Dp was applied because it is a three-electrode array similar to the MAN array, and the $\gamma_{11 \mathrm{n}}$ arrays themselves are getting closer and closer to be threeelectrode arrays with the increasing number of $n$. Wenner$\alpha$ configuration was chosen because it is one of the most popular and best known configurations, whereas the Stummer configuration (Stummer et al. 2004) should have to be the best conventional configuration because it was constructed using an optimization process. By comparing the $\gamma_{11 \mathrm{n}}$ and $\gamma_{\mathrm{m} 11 \mathrm{n}}$ results with the results of these configurations, one can therefore obtain an oversight about their abilities.

In Fig. 6 , the results of $\gamma_{11 n}$ configurations only for $n=1-4$ are shown, which gives satisfactory information. In Figs. 8 and 9, at the same time, the whole series of $\gamma_{11 \mathrm{n}}$ configurations $(n=1-7)$ is presented to have an oversight about all these configurations. $n$ is limited to 7 because its further increase leads to too less data points. $m$ was $1-14$, $1-11,1-9,1-7,1-7$, and $1-7$ for the $\gamma_{11 \mathrm{n}}$ configurations for $n=2-7$ (Fig. 1), accordingly.

The parameters for the traditional configurations are shown in Fig. 3. The configurations used in the optimized Stummer configuration can be found in (Stummer et al. 2004).

The data coverage and number of data points are shown in Fig. 3 for the traditional configuration and in Fig. 4 for the $\gamma_{11 \mathrm{n}}, \gamma_{\mathrm{n} 11}$, and $\gamma \mathrm{m}_{11 \mathrm{n}}$ configurations $(\mathrm{n}=2-7)$, While the Wenner- $\alpha$ and $\gamma_{111}$ (Wenner- $\gamma$ ) configurations have only 570 data points, the Stummer configuration has 669 , the Dp-Dp 

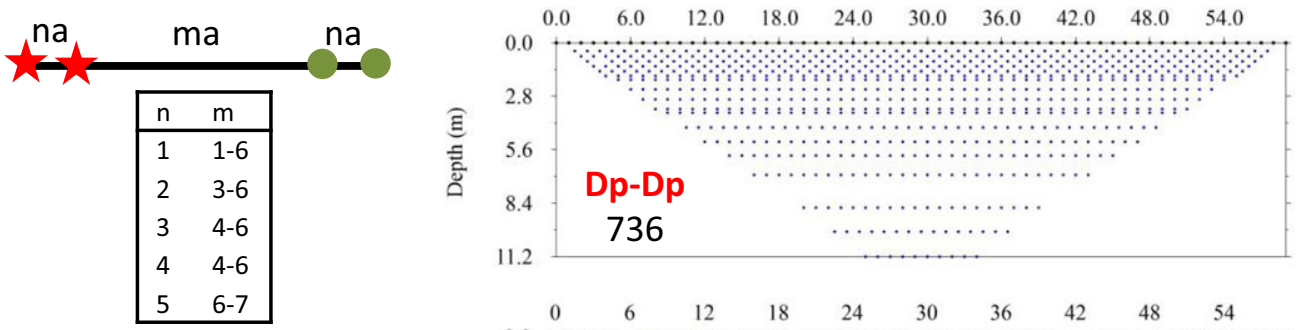

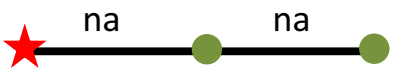

$n=1-28$

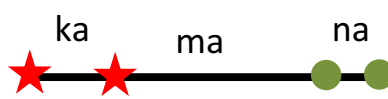

For the parameters see Stummer et al. 2004.

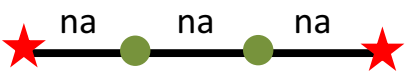

$n=1-19$

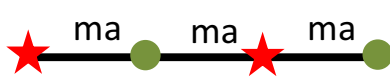

$n=1-19$
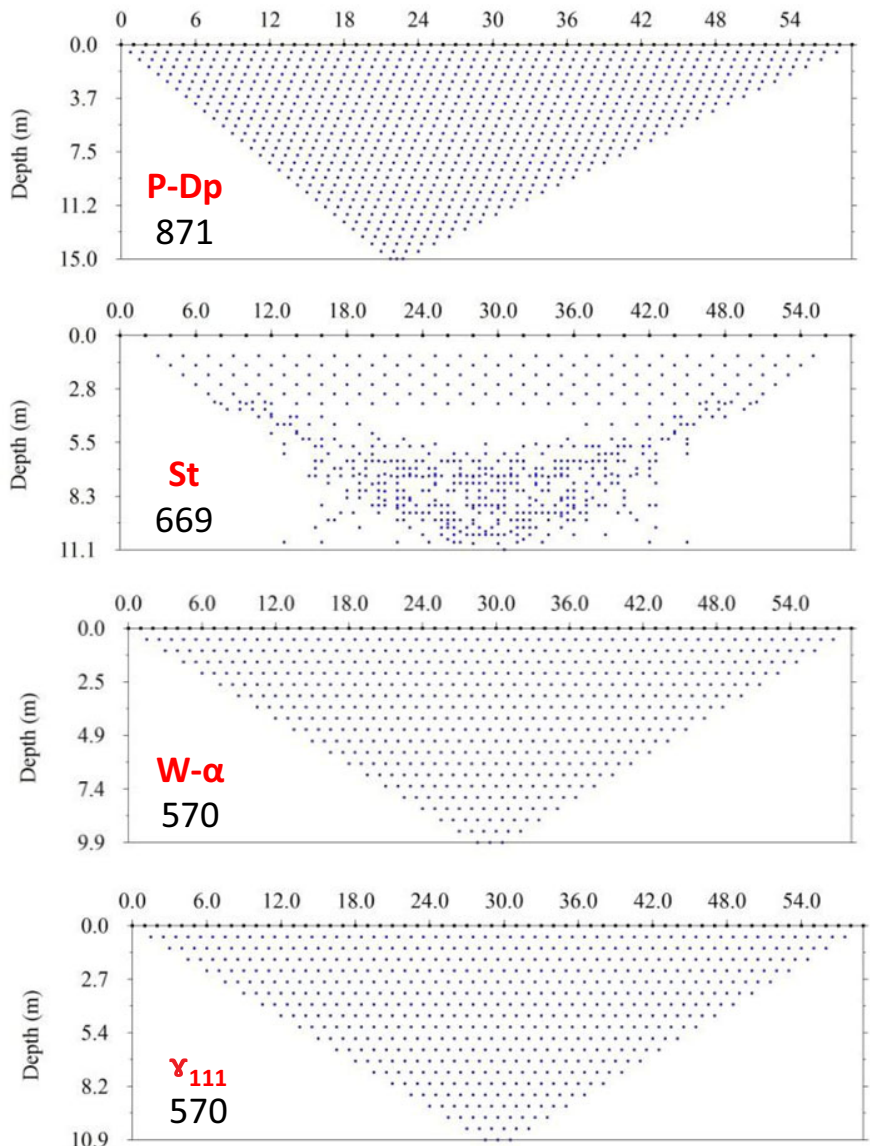

Figure 3 Left side: the applied traditional configurations with their parameters. Stars denote current electrodes, and full circles denote potential electrodes. Right side: Data coverage and number of data points for the same configurations.

configuration has 736, and the P-Dp configuration has 871 . In contrary to these configurations, the $\gamma_{11 \mathrm{n}}$ configurations (Fig. 3) have no more than 420 data points, i.e., their measuring time is significantly less than those of the traditional configurations. When $n$ increases, the number of data points is even decreasing drastically. The mirrored version of the $\gamma_{11 \text { n }}$ configurations contain two times as many point as its original version, but even in this case, the number of the data points are only $840,660,540,448,392$, and 342 for $n=2-7$, accordingly. It means that, disregarding the $\gamma \mathrm{m}_{112}$ configuration, even the mirrored configurations have less data points, i.e., their measuring time is shorter than that of the Stummer,
Dp-Dp, and P-Dp configurations (which used to produce the best results among the traditional configurations, according to Szalai et al. 2013a).

\section{Inversion parameters}

All numerical calculations presented in this article were carried out by EarthImager, Version 2.1.6 (EarthImager 2006). The parameters that are different from the software's basic parameters are summarized in Table 1 . The basic parameters were only changed if it was necessary to get reasonable results. For example, the negative resistivity 

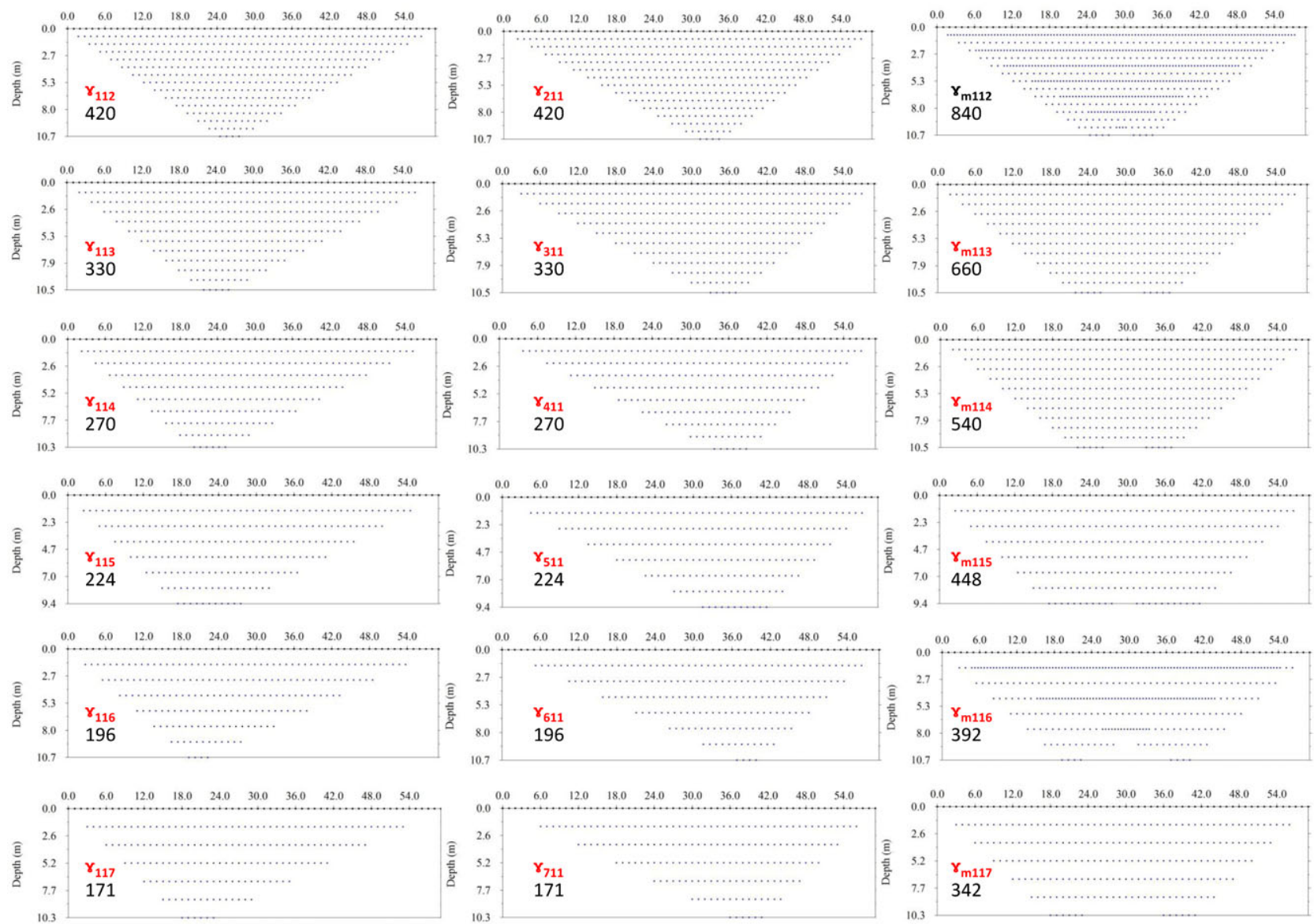

Figure 4 Data coverage and number of data points for the $\gamma_{11 \mathrm{n}}, \gamma_{\mathrm{n} 11}$, and $\gamma \mathrm{m}_{11 \mathrm{n}}$ configurations $(\mathrm{n}=2-7)$.

values of the Minimum Apparent Resistivity parameters were selected because the signal may change its sign. To create Fig. 6, Pseudosection was applied in the inversion process as Starting Model, i.e., the section that contains the "measured" data. In all inversion processes, $1 \%$ Gaussian noise was added to the data (with the exclusion of the inversion whose results are presented in Fig. 6), and root mean square (RMS) and L2 norm were used to study the data misfit. L2 norm is defined as the sum of the squared weighted data errors (difference between predicted/calculated resistivity and observed/measured resistivity). The RMS error is its normalized version that also takes into account the data number.

The finite-element method (FEM) was used in the modeling, which is a numerical technique for finding approximate solutions to boundary value problems for differential equations. It uses variational methods to minimize an error function and produce a stable solution. FEM connects many simple element equations over many small subdomains, named finite elements, to approximate a more complex equation over a larger domain. FEM produces more accurate forward modeling solution than the finite-difference method.

In many cases, it would be possible to get better results than the ones presented by taking the images from other iteration steps. The selection of the most appropriate inverted section requires however knowledge of the model (which was used to calculate synthetic data) because the decrease in the RMS does not inevitably result in better images. In field measurements, this knowledge is certainly not available, although the final aim of such studies (including numerical investigations) is to check the applicability of different configurations in the field. Therefore, field data processing requires a more or less automatic inversion. This is why Stop RMS error option was activated (values shown in Table 1), which completed the inversion in many cases after the first or second iteration. It was also important to apply the same inversion parameters to all configurations to get (automatic) objective results, making it possible to compare them. 

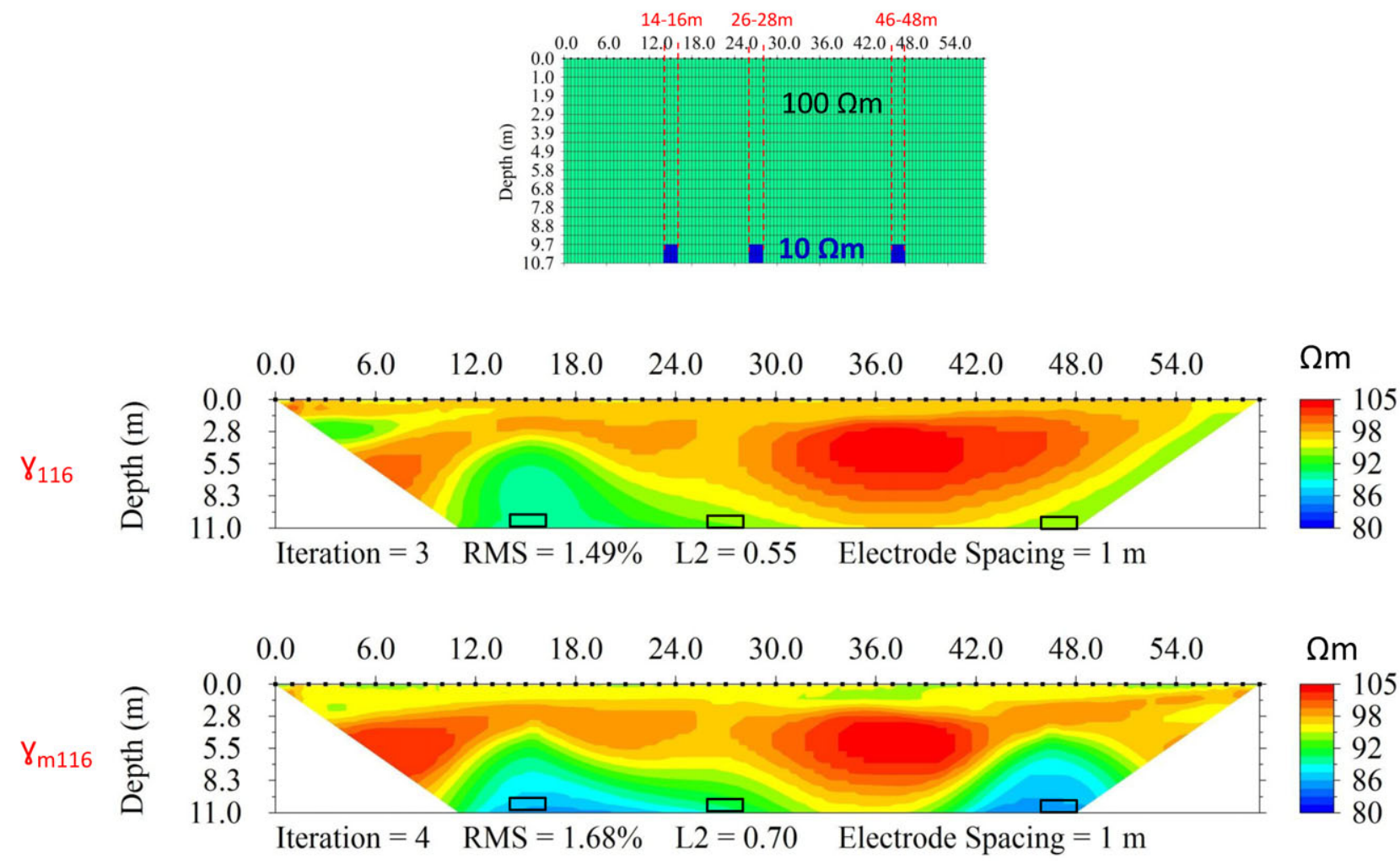

Figure 5 Example to show the advantage of the mirrored configurations against the single ones. The model is given on the top of the figure. The resistivity of the prisms is $10 \Omega \mathrm{m}$, whereas the half-space resistivity is $100 \Omega \mathrm{m}$.

RMS and L2 may not always be adequate to estimate the image quality. Often, images with smaller (that is better) RMS and L2 proved to be worse because they contained more pseudo-anomalies and more significant (therefore more disturbing) ones, and the shape of the anomaly was also further different from the model. The RMS and L2 are severely influenced by the resistivity values, and they may not be as sensitive to the geometrical parameters of the anomaly, which is often more important. Therefore, we prefer to qualify the inverted image obtained from numerical investigations similarly to that presented in "The criteria to interpret the results" section.

For many $\gamma(\mathrm{m})_{11 \mathrm{n}}$ configurations and for many models, RMS was proved to be high. There are two main reasons.

- The denominator $d_{i}$ Meas in the RMS function may be very small for many data, even close to zero due to that the signal may change its sign (Szalai et al. 2004). It leads to very large values for individual measurements, and therefore their sum, i.e., the RMS, may also be large.

- The numerator may also be large for the same data point due to the rapid changes of the signal close to these small values. In a theoretical case, if the predicted and measured curves (for a given depth) would be the same but would be slightly shifted horizontally from each other, one would get very large RMS. This is not the case for the traditional configurations where the horizontal gradient of the signal is much smaller.

RMS and L2 are therefore not always appropriate values to estimate the quality of $\gamma(\mathrm{m})_{11 \mathrm{n}}$ measurements. Another value should be found to quantitatively estimate the quality of $\gamma(\mathrm{m})_{11 \mathrm{n}}$.

In the present article, several models were numerically studied that aimed to illustrate: (i) the effect of the resistivity contrast to the inverted image, (ii) the horizontal and (iii) vertical resolution capacity of the different configurations, (iv) the applicability of these configurations for larger effect anomalies, and (v) the applicability for a realistic model.

\section{The criteria to interpret the results}

In the qualification and comparison of the results obtained by different configurations, the main point (point 1a) is whether the model body can be seen, i.e., whether is there an anomaly where the body should appear. Only if there is an anomaly will there be a sense to continue the interpretation. In this 


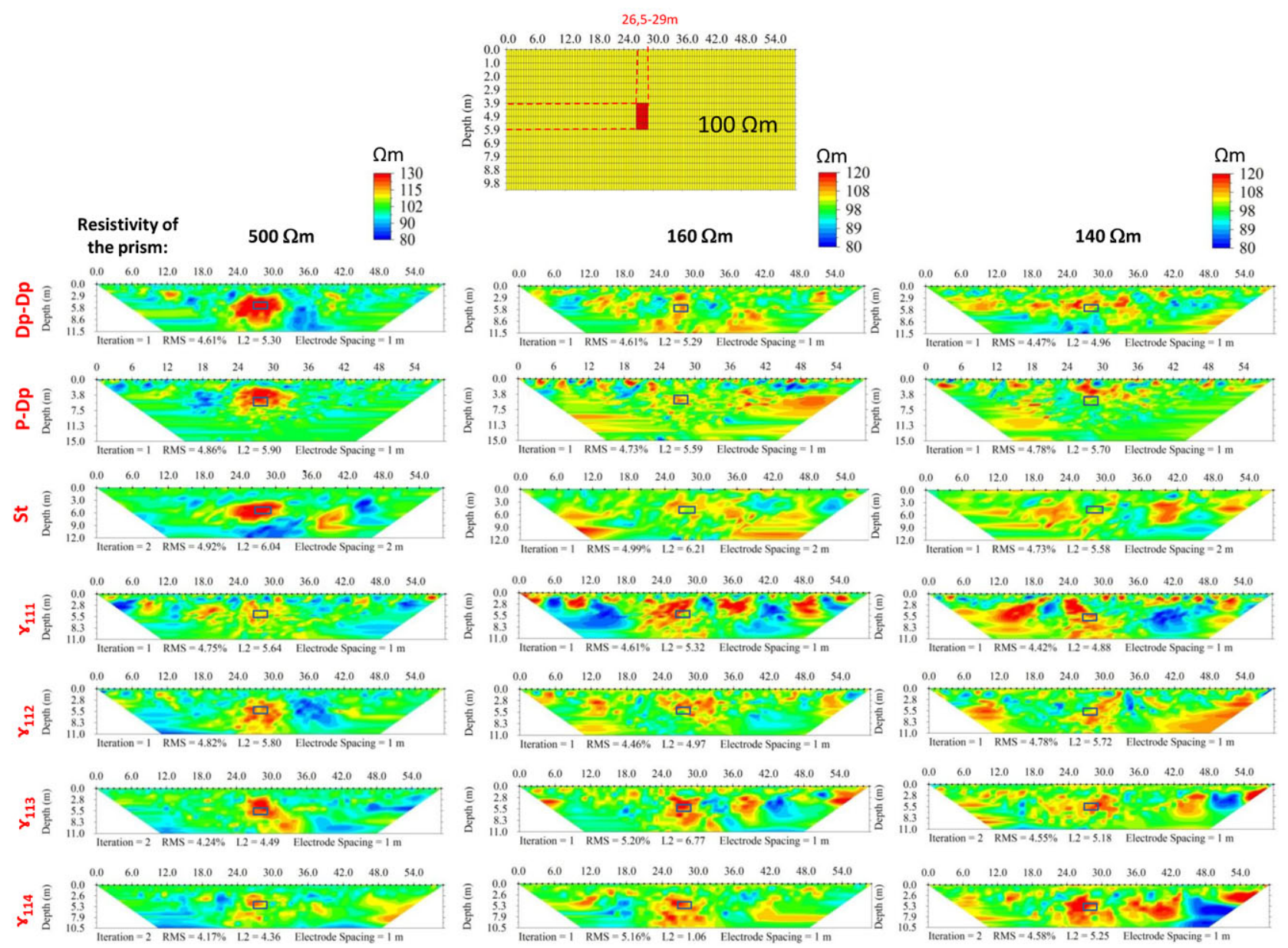

Figure 6 Inverted sections for several traditional and $\gamma_{11 \mathrm{n}}$ configurations. Resistivity values of the prisms are $500 \Omega \mathrm{m}, 160 \Omega \mathrm{m}$, and $140 \Omega \mathrm{m}$. Background resistivity is $100 \Omega \mathrm{m}$. The model is given on the top of the figure.

case, the next point (Fig. 1b) is whether there are any other (so-called pseudo) anomalies that are not awaited to be there, and how much they influence the interpretation. The larger they are in their extension and/or in their resistivity contrast to the background value, the more they can mislead the interpretation. If the body is detectable in the following, the horizontal (Fig. 2a) and vertical (Fig. 2b) positions of the anomaly, its size (Fig. 2c), its resistivity value (Fig. 2d), and, in an ideal case, its shape (Fig. 2e) can be compared with the model parameters and be taken into account in the interpretation.

In our investigations, however, where the aim was to investigate small-impact model bodies, the principal question had to be whether the anomaly due to the model will at least appear on the inverted image. This was the case, e.g., in Fig. 6. In this case, if there are more model bodies, their separability (3) can be an important item. This is the case in the resolution investigations in Figs. 8-10. If the bodies are separated from each other in the inverted image, the same questions (Fig. 1a, $1 \mathrm{~b}$, and $2 \mathrm{a}-\mathrm{e}) \mathrm{can}$ be regarded as single bodies.

\section{Results of the numerical investigations and their interpretation}

Before going into the details of the numerical simulations, we explain why $\gamma \mathrm{m}_{11 \mathrm{n}}$ configuration results will be presented instead of or in addition to $\gamma_{11 \mathrm{n}}$ configurations. Figure 5 shows $\gamma_{116^{-}}$and $\gamma \mathrm{m}_{116}$ images for a model containing three conductive prisms. Their resistivity values are $10 \Omega \mathrm{m}$ in the $100-\Omega \mathrm{m}$ resistivity half-space. It is easy to see that the $\gamma \mathrm{m}_{116}$ configuration is able to separate the prisms from each other better than the $\gamma_{116}$ configuration by itself, especially the prisms in the right side, which are separated from each other very well. The effect of the prism in the middle of the section is also more remarkable in the $\gamma \mathrm{m}_{116}$ image. 
Resistivity of the prism

$160 \Omega m$

P-Dp
Dp-Dp

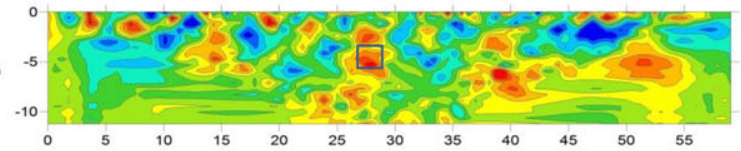

$\gamma 112$

$\gamma 113$

$\gamma 114$

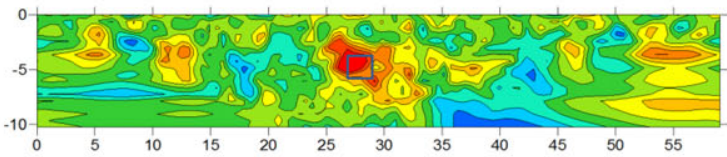

P-Dp

Dp-Dp

$\gamma 112$

$\gamma 113$

$\gamma 114$

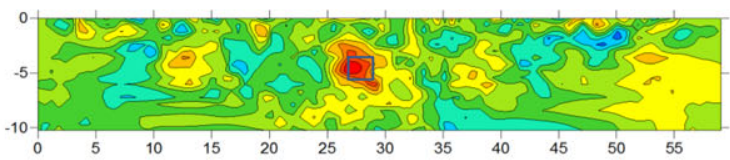

$140 \Omega m$

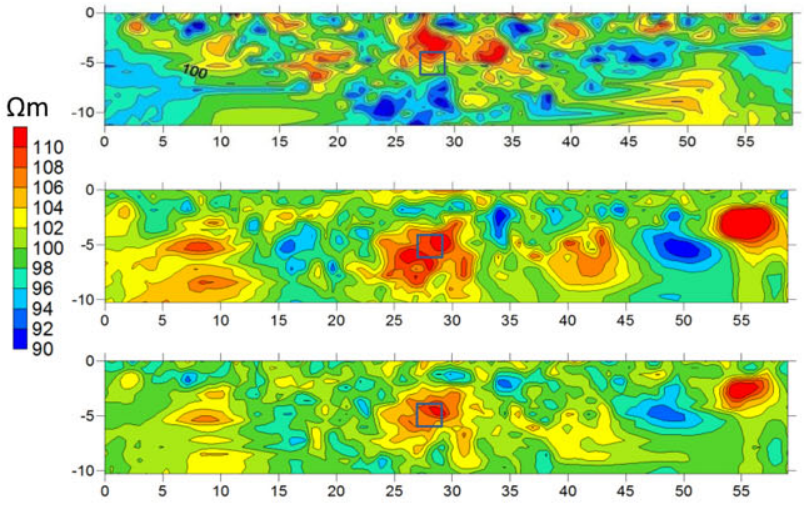

Figure 7 MOST images of different configuration combinations.

Investigations of further models not presented here motivated us to use the $\gamma \mathrm{m}_{11 \mathrm{n}}$ configurations. They produced better images than the $\gamma_{116}$ configurations, especially if the inhomogeneity is in the deeper parts of the model. This is the reason we prefer to use the mirrored version of these configurations. In the present stage of the investigations, it seems, however, to be reasonable to present also the $\gamma_{116}$ results, at least for a few models.

The results of DD investigations by Szalai et al. (2013b) and MAN configuration studies (Szalai et al. 2004) referred to the usefulness of the $\gamma_{11 n}$ configurations, particularly if the effect of the inhomogeneity is small, i.e., if its size/depth ratio and/or its resistivity contrast to the host is small. At first, the image of a small size prism will be compared with those of the often applied dipole-dipole (Dp-Dp), pole-dipole (P-Dp), optimized Stummer (St), and $\gamma_{11 \mathrm{n}}(n=1-4)$ configuration's images (Fig. 6).

While the resistivity of the host in Fig. 6 was $100 \Omega \mathrm{m}$, the resistivity values of the prism were $500 \Omega \mathrm{m}, 160 \Omega \mathrm{m}$, and $140 \Omega \mathrm{m}$ in the columns in Fig. 6 . The depth of the upper side of the prism is $3.9 \mathrm{~m}$, its thickness is $2.0 \mathrm{~m}$, and its horizontal extension is $2.5 \mathrm{~m}$, between 26.5 and $29 \mathrm{~m}$. For the applied inversion parameters, see Table 1.

If the resistivity of the prism was $500 \Omega \mathrm{m}$ (Fig. 6, left column), i.e., the resistivity contrast to the background was significant, the prism proved to be detectable by each configurations; however, the application of the $\gamma_{111}$ configuration is not suggested due to the significant artefacts that are in the same resistivity range than the "real" anomaly itself. The other configurations detect the model clearly, they position it correctly, and the pseudo-anomalies were not comparable to the real anomaly. The size of the anomaly is, however, larger than expected.

The conventional configurations proved to be better for this model because the effect of the anomaly is large due to the large resistivity contrast between the model and the background. For such models, they work properly, whereas the inversion of the $\gamma(\mathrm{m})_{11 \mathrm{n}}$ configuration data for large effect inhomogeneity is not well resolved yet.

For the $160-\Omega \mathrm{m}$ prism (Fig. 6, middle column) only the $\gamma_{113^{-}}$, and $\gamma_{114}$ configuration images are somewhat convincing, whereas the traditional configurations proved to be rather ineffective. The $\gamma_{113}$, and $\gamma_{114}$ images present a resistive anomaly at the right position, which arises quite characteristically from the background, and the artefacts are smaller in their size than the anomaly. The $\gamma_{114}$ configuration results are the most convincing from the whole series, although the anomaly is not at all sharp in its case, neither.

Finally the $140-\Omega \mathrm{m}$ prism (Fig. 6, right column) can be detected by all configurations, excluding the Stummer one, but the anomalies were more or less mispositioned. The $\gamma_{113}$ and $\gamma_{114}$ configurations seem to be the closest to the real model, although they also produced significant artefacts. The most important is however the presence of an anomaly at the position of the inhomogeneity that is inevitable for a correct interpretation.

Artefacts could nevertheless easily mislead the interpretation of field data. To avoid misinterpretation, however, there are several possibilities. If the location of the target is more or less known, even images with artefacts enable its more precise localization and description. In this case, the artefacts should not be taken into account. If there may be more prism-like 

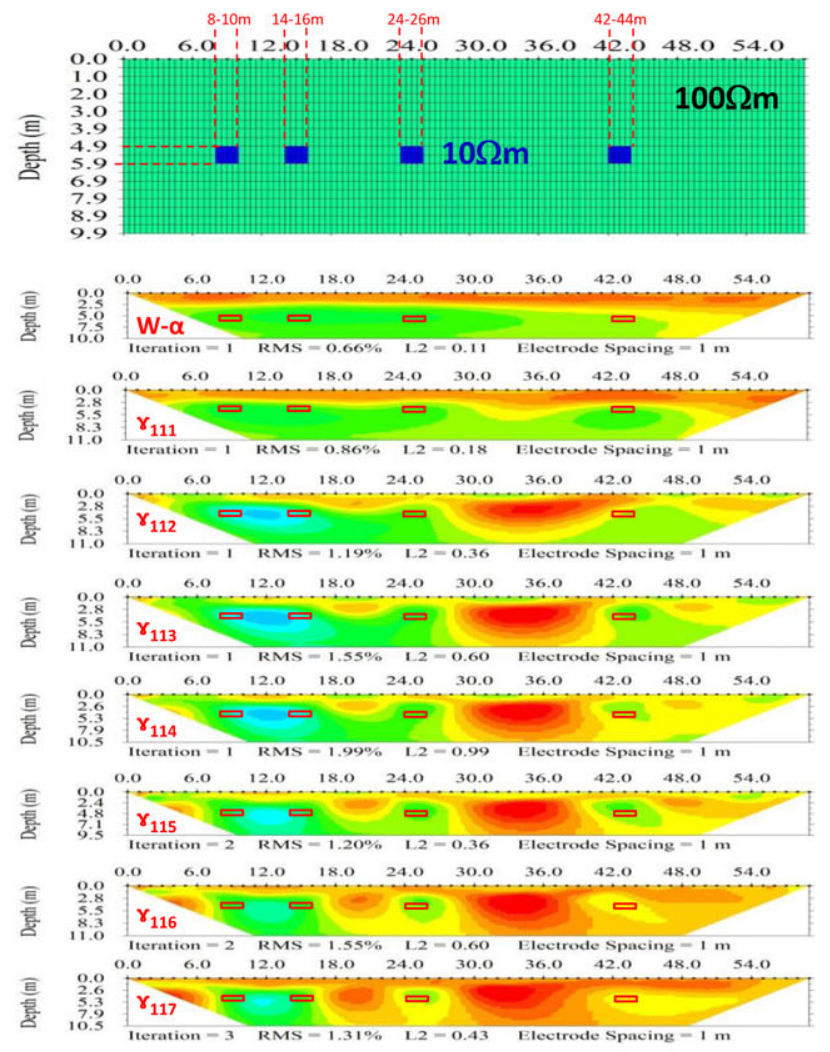
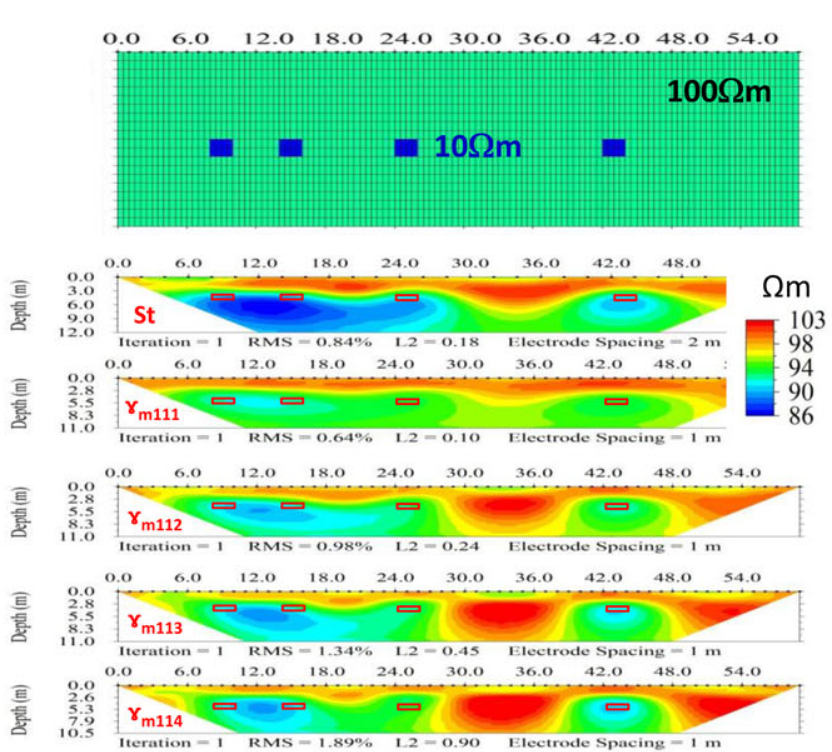

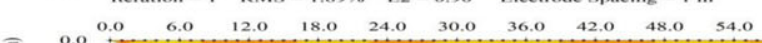

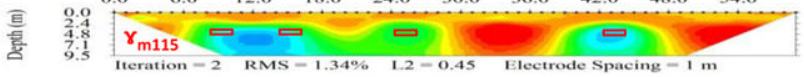

$\begin{array}{lllllllllllll}0.0 & 0.0 & 6.0 & 12.0 & 18.0 & 24.0 & 30.0 & 36.0 & 42.0 & 48.0 & 54.0\end{array}$

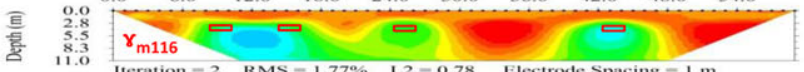

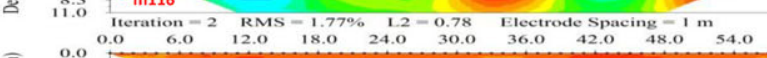

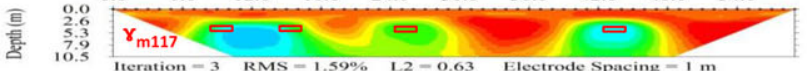

Figure 8 Horizontal resolution investigation for different traditional and $\gamma_{11 \mathrm{n}}$ configurations. The model is given in the first row.

objects and there is not any information regarding their position, it is possible to first compare the data of different geoelectric configurations. If images of many configurations display an anomaly at the same position, it is highly probable that there is inhomogeneity. If an anomaly appears on only one or two images, it is most likely an artefact. An anomaly with a large value and extension (e.g., on the $\gamma_{114}$ image in the right column between about $34 \mathrm{~m}$ and $44 \mathrm{~m}$ ) does not have any pair on the $\gamma_{112}$ or $\gamma_{113}$ images. Therefore its validity is strongly questioned. In contrast, the anomaly in the middle of the section appears on all of these images (although not exactly at the same position), increasing the probability of the existence of inhomogeneity there. The model stacking (MOST) algorithm (Leontarakis and Apostolopoulos 2012, 2013) basically uses the same principle. In this process, artefacts due to random noises eliminate each other, whereas the anomalies due to real objects strengthen each other (see Fig. 7, discussed later in more detail). Second, similar procedures can be applied, carrying out measurements several times with the same configuration (stacking). Thirds, the comparison of geoelectric results with the results of other geophysical measurements or the joint inversion of different data sets could also decrease the uncertainty. Fourth, A direct investigation at the problematic places is also possible through excavations or boreholes. Which of these procedures is applied is a question of money.

While for the models with high resistivity contrast (500 $\Omega \mathrm{m}$ ), the traditional configurations proved to be better; their quality decreases faster with the decreasing resistivity contrast than that of the $\gamma_{11 \mathrm{n}}$ configurations. In the small resistivity contrast range, the application of the $\gamma_{11 n}$ configurations seem to be more worthwhile, and the quality of their image can be further improved by the MOST procedure introduced by Leontarakis and Apostolopoulos (2012, 2013). By stacking the models of different configurations, the model of the combined configuration leads to a final model almost free of artefacts with extremely high resolution in shape and positioning, and an intense representation of the targets. The MOST procedure is based on a simple statistical approach, calculating the geometric mean of the different values, which are given by each model for the same point of the half-space (Leontarakis and Apostolopoulos, 2012, 2013). 

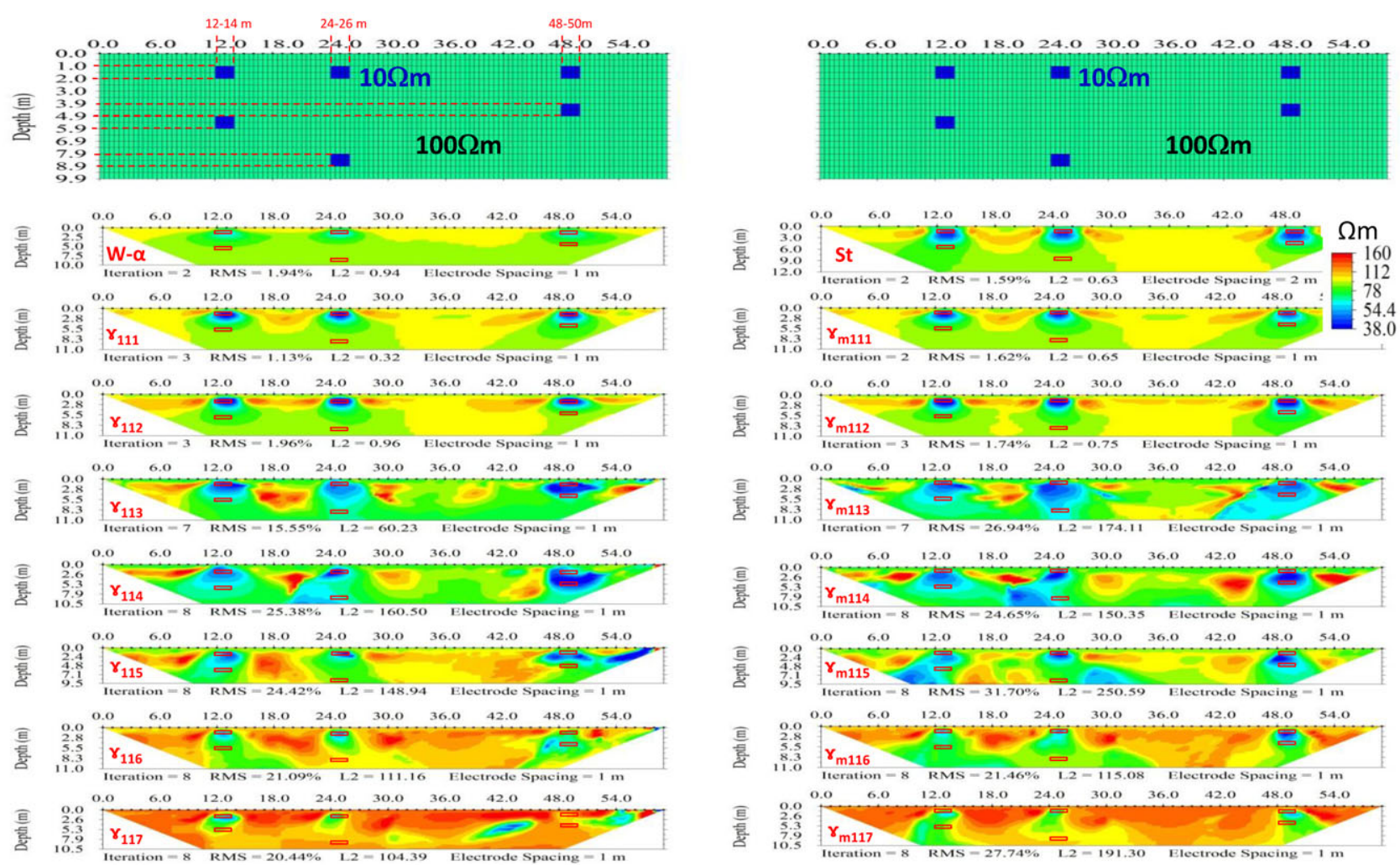

Figure 9 Vertical resolution investigation for different traditional and $\gamma_{11 \mathrm{n}}$ configurations. The model is given in the first row.

The RMS error values for the images in Fig. 6 were between $4.2 \%$ and $5.2 \%$ for all configurations, i.e., in this sense, there was no significant difference among the investigated configurations.

Figure 7 presents the effect of the MOST procedure. In the first row in Figure 7, the MOST results made of the combination of the P-Dp and Dp-Dp configuration results are shown for both the $160-\Omega \mathrm{m}$ and $140-\Omega \mathrm{m}$ prism models. This configuration combination contains 1540 data points. For the $160-\Omega \mathrm{m}$ prism, the MOST result is more convincing than the results of the simultaneous configurations (Fig. 6), but it still contains a lot of artefacts. The MOST result made of the $\gamma_{112}, \gamma_{113}$, and $\gamma_{114}$ configurations (Fig. 7, second row) is much more convincing, in spite of the fact that this combination contains only about 30\% less 1020 data points. The resistivity values of the artefacts are in this case not comparable with that of the real anomaly, and it became sharper and more characterized than in the individual images (Fig. 6). The quality of the image could be even further improved by stacking all of these configurations (Fig. 7, third row), but the measurement thus becomes less economic.
The situation is about the same for the $140-\Omega \mathrm{m}$ prism model (Fig. 7, right column). The MOST procedure led to reasonable image for both (traditional-, and $\gamma_{11 \mathrm{n}^{-}}$) configuration combinations. In this case, even the combination of all configurations (Fig. 7, third row) seems to be reasonable if the aim is to get high-quality image even among very wrong conditions. Disregarding the artefacts at the end of the profile, the prism was clearly detected in this way, and all its geometrical parameters that are its horizontal and vertical positions and even its size are satisfactory.

If artefacts do not disappear even after carrying out the MOST procedure, it is still possible to use other geophysical techniques or apply direct procedures to decrease the uncertanity of the interpretation.

Summarizing the results of Figures 6 and 7, it can be stated that although the traditional Dp-Dp and Stummer configurations proved to be well usable if the resistivity contrast was larger $(500 \Omega \mathrm{m})$, the $\gamma_{11 \mathrm{n}}$ configurations proved to be more and more fruitful in comparison with the traditional configurations if the contrast was smaller $(160,140 \Omega \mathrm{m})$. The advantageous features of the $\gamma_{11 \mathrm{n}}$ configurations became especially spectacular by combining them using the MOST 
Table 1 The parameters applied in the numerical investigations that are different from the basic (Surface) parameters of the EarthImager v2.1.6. software. The parameters different from the basic ones are written in bold.

\begin{tabular}{|c|c|c|c|}
\hline & Surface & Fig. 6. & Fig. 8-11. \\
\hline Minimum apparent resistivity $(\Omega \mathrm{m})=$ & 1 & -100000000 & -10000 \\
\hline Maximum apparent resistivity $(\Omega \mathrm{m})=$ & 10000 & 1000000 & 10000 \\
\hline Keep All Data: & No & Yes & No \\
\hline Lower-layer-thickness/Upper-layer-thickness $=$ & 1.1 & 1 & 1 \\
\hline Depth of inverted Model/Depth of Pseudosection $=$ & 1.1 & 1 & 1 \\
\hline Max number of iteration of nonlinear inversion $=$ & 8 & 20 & 8 \\
\hline Stop RMS error $=$ & $3 \%$ & $5 \%$ & $2 \%$ \\
\hline Stop when L2 norm is small enough: & No & Yes & No \\
\hline Initial Lagrange multiplier or roughness factor $=$ & 10 & 1 & 100 \\
\hline Starting model: & Avg App Res & Pseudosection & Avg App Res \\
\hline Estimated noise of resistivity data $=$ & $3 \%$ & $2 \%$ & $2 \%$ \\
\hline Initial damping factor of resistivity $=$ & 10 & 1 & 100 \\
\hline
\end{tabular}

procedure. The application of the $\gamma_{11 \mathrm{n}}$ configurations and of the MOST procedure is therefore highly recommended in case that a small impact anomaly should be found in a noisy environment.

In the proceeding, figures only for Wenner- $\alpha$ and Stummer configuration results will be shown from the traditional ones because we wanted to compare our results with the results of a popular traditional configuration (W- $\alpha$ ) and with that of the best traditional configuration $(\mathrm{St})$. At this stage of the study, we found an important to present also the $\gamma_{11 \mathrm{n}}$ configuration results beside of the $\gamma \mathrm{m}_{11 \mathrm{n}}$ configuration ones.

Figure 8 presents the horizontal resolution capacity of the Wenner- $\alpha$, Stummer-, $\gamma_{11 \mathrm{n}^{-}}$, and $\gamma \mathrm{m}_{11 \mathrm{n}}(n=1-7)$ configurations. For the applied inversion parameters, see Table 1 . The model parameters are presented on the top of Fig. 8 . The Wenner- $\alpha$ configuration was unable to separate the conductive prisms from each other. The Stummer configuration clearly separates the right-hand prism from the others, and the separateness of the second prism from the right side may be also supposed. The $\gamma_{11 \mathrm{n}^{-}}$, and $\gamma \mathrm{m}_{11 \mathrm{n}}$ configurations (from $n=2$ ) separate the prism farthest on the right from the others even more convincingly creating a high-resistivity region (29 $\mathrm{m}-38 \mathrm{~m}$ ) between the prisms. With increasing $n$ the second prism from the right side separates itself more convincingly from the other prisms (there is again a high-resistivity zone between $18 \mathrm{~m}$ and $22 \mathrm{~m}$ ). The first two bodies in the left side-whose distance is comparable to their depth (4 m versus $4.9 \mathrm{~m}$ )—could not have been separated from each other by neither of the studied configurations. From the point of view of their horizontal resolution capacity, both the $\gamma \mathrm{m}_{11 \mathrm{n}}$, and the $\gamma_{11 \text { n }}$ configurations proved to be definitely better than the optimized Stummer configuration, not speaking about the
Wenner- $\alpha$ configuration. The RMS value was below $2 \%$ for each configurations. Figure 9 demonstrates the results of vertical resolution investigations for the same configurations. For the applied inversion parameters, see Table 1 . The model parameters are given on the top part of Figure 9. All prisms closer to the surface were detected by each configuration. The Stummer and $\gamma(\mathrm{m})_{11 \mathrm{n}}(n=1,2)$ configurations proved to be almost perfect regarding all quality parameters. The near-surface anomalies of the W- $\alpha$ and the other $\gamma(\mathrm{m})_{11 \mathrm{n}}$ configurations are not as sharply delineated, but they are also satisfactory.

For the prisms on deeper levels, only the one on the right side was observed by all configurations (excluding only the $\gamma_{117}$ configuration), but it merged into the one above it because they are too close to each other

From the prism pair on the left side, the deeper one proved to be almost undetectable even by the Stummer configuration. In contrary, most $\gamma(\mathrm{m})_{11 \mathrm{n}}$ results refer to the existence of the deeper prism. They show a long, narrow anomaly downwards (e.g., $\gamma_{115}, \gamma_{116}$, and $\gamma \mathrm{m}_{117}$ configurations) or even an anomaly that delineates well the prism pair below each other (e.g., $\gamma_{117}, \gamma \mathrm{m}_{113}$, and $\gamma \mathrm{m}_{114}$ configurations). The $\mathrm{W}-\alpha$ configuration indicates the deeper anomaly but with a very wide and uncertain anomaly.

The Stummer configuration indicates weakly the existence of the deeper prism in the middle of the section, but the anomalies produced by the $\gamma_{113}, \gamma_{116}, \gamma \mathrm{m}_{116}, \gamma \mathrm{m}_{117}$ configurations and especially that of the $\gamma \mathrm{m}_{113}$ configuration are more convincing. Their anomalies are narrower and/or get closer to the depth of the deeper prism, and/or the values of the anomalies differ more from the background value. 

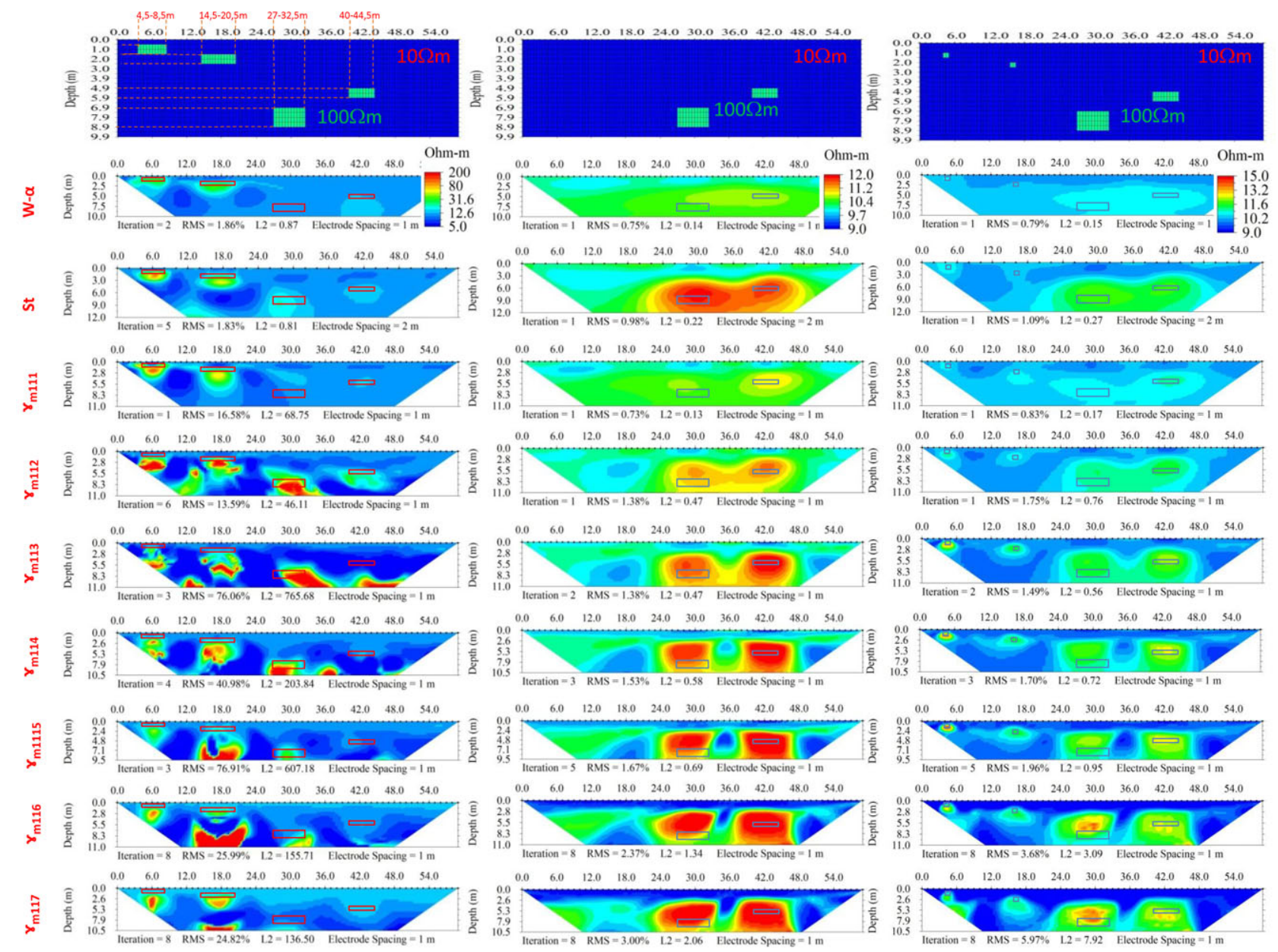

$\begin{array}{llllllllll}0.0 & 6.0 & 12.0 & 18.0 & 24.0 & 30.0 & 36.0 & 42.0 & 48.0 & 54.0\end{array}$
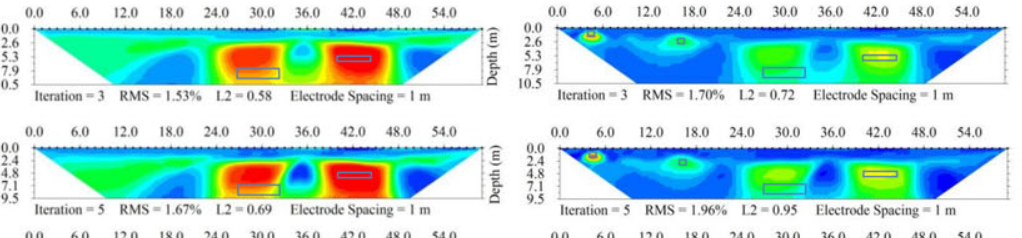

$\begin{array}{llllllllll}0.0 & 6.0 & 12.0 & 18.0 & 24.0 & 30.0 & 36.0 & 42.0 & 48.0 & 54 .\end{array}$

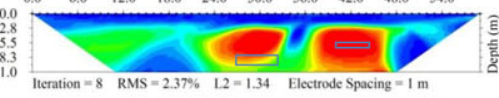

$\begin{array}{llllllllll}0.0 & 6.0 & 12.0 & 18.0 & 24.0 & 30.0 & 36.0 & 42.0 & 48.0 & 54.0\end{array}$

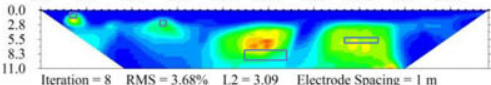

$\begin{array}{llllllllll}0.0 & 6.0 & 12.0 & 18.0 & 24.0 & 30.0 & 36.0 & 42.0 & 48.0 & 54.0\end{array}$
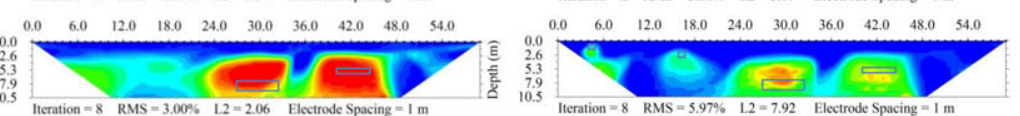

Figure 10 Left column: Inversion results from the Wenner- $\gamma$, Stummer and $\gamma(\mathrm{m})_{11 \mathrm{n}}(n=1-7)$ configurations for the model similar to that in (Wilkinson et al. 2006). Middle column: Results for the same model without the near-surface anomalous bodies. Right column: The first model with smaller near-surface inhomogeneities. The model is given in the first row.

For this model, the RMS values of the $\gamma_{11 \mathrm{n}}-\gamma / \mathrm{m}_{11 \mathrm{n}}$ configurations from $n=3$ were systematically much larger $(20 \%-$ $32 \%$ ) than those of the traditional configurations (below $2 \%$ ). As it has however already been mentioned, the principal question is the similarity of the inverted image to the reality, which is the model in numerical investigations. RMS value seems to increase with increasing $n$ in this model. It may happen because, with increasing $n$, the arrays approach the null array situation and produce sharper and sharper anomalies.

The separation of the prisms below each other was impossible for all the configurations we studied, but certain $\gamma_{11 \mathrm{n}}{ }^{-}$and $\gamma \mathrm{m}_{11 \mathrm{n}}$ configurations, particularly the $\gamma \mathrm{m}_{113}$ configuration, proved to be better in detecting the deeper bodies.

Next, we investigated a model that did not seem to be favourable for the $\gamma \mathrm{m}_{11 \mathrm{n}}$ configurations (Fig. 10) because of the large size of the inhomogeneity. (Inversion parameters are in Table 1)

The model in the left column of Fig. 10 is very similar to the one studied by Wilkinson et al. (2006). Here the anomalous bodies with large resistivity contrast $(100 \Omega \mathrm{m}$ in comparison to the $10-\Omega \mathrm{m}$ half-space value) and the large size can be seen better in the Stummer image than in most $\gamma \mathrm{m}_{11 \mathrm{n}}$ configuration images. Although the deepest (from the detectability point of view) most critical body is displayed more convincingly by the $\gamma \mathrm{m}_{11 \mathrm{n}}(\mathrm{n}=2-6)$ configurations, these images contain several pseudo-anomalies, as well. In such a case again, a solution similar to the one applied by Leontarakis and Apostolopoulos (2012, 2013) could be suggested to supress the pseudoanomalies and highlight the real anomalies. We would like to call your attention also to the $\gamma \mathrm{m}_{112}$ configuration that gives-in spite of its higher RMS 


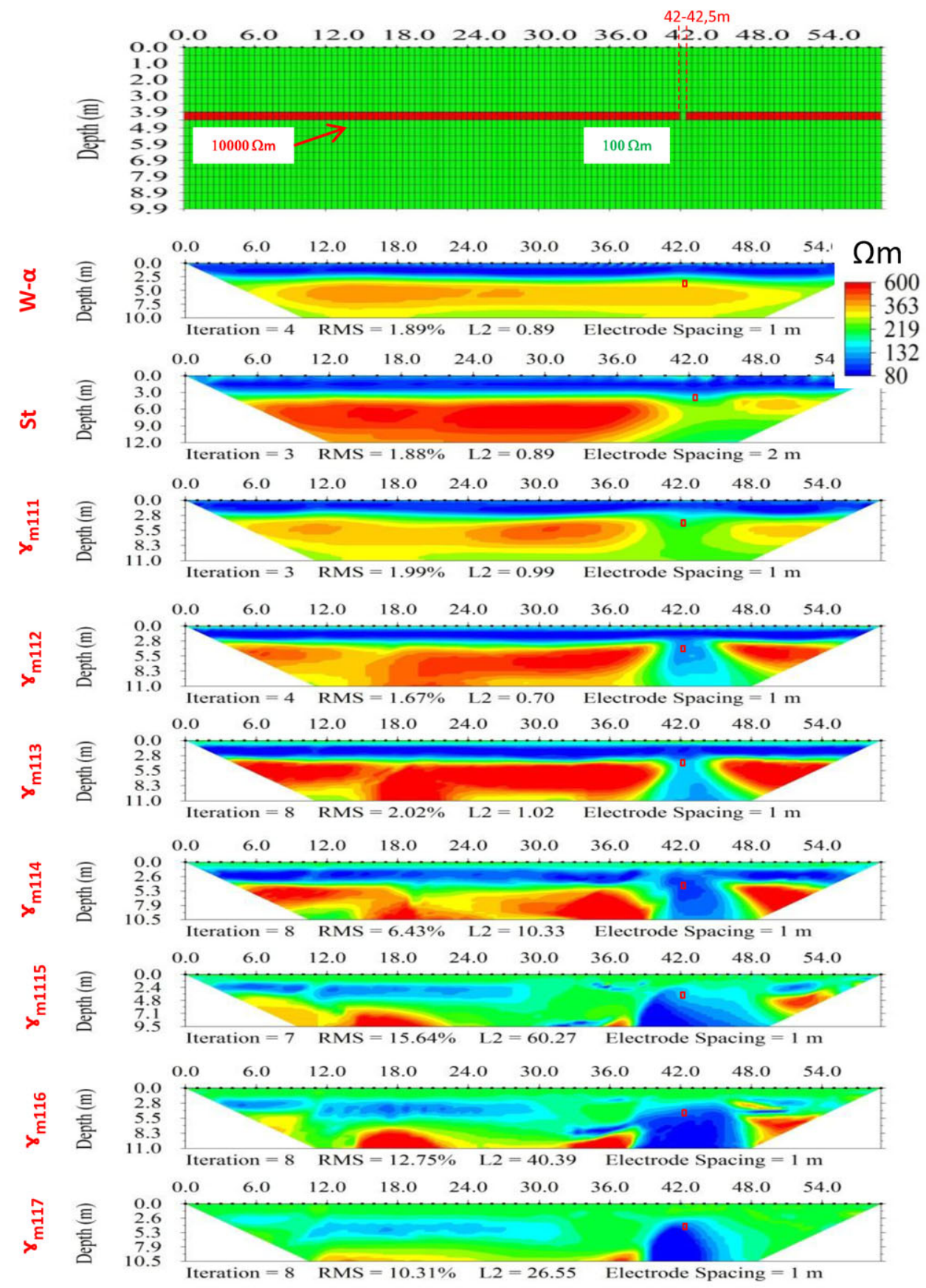

Figure 11 The effect of a hole in the liner at the bottom of a waste deposit from different configurations. The uppermost figure is the model we investigated.

value-the most characteristic image of the prisms, disregarding the pseudo-anomaly in the left bottom part of the section.

If, however, the near-surface bodies are not present (Fig. 10, middle column), all $\gamma \mathrm{m}_{11 \mathrm{n}}$ configurations give better results than the Stummer configuration. They can separate the two deeper bodies from each other more impressively. It is especially true if $n$ is at least 3 . The $\gamma \mathrm{m}_{11 \mathrm{n}}$ images remain as impressive even in the presence of near-surface bodies if they do not influence to the surface potential too much (Fig. 10, right column). It is remarkable that also the near-surface 
bodies are presented more convincingly by the $\gamma_{11 n^{-}}$than by the traditional configurations. The $\gamma \mathrm{m}_{116}$ configuration produced the best image that resembles the best to the model. It separated all prisms unambiguously from each other; their horizontal positioning was perfect, and their vertical positioning was reasonable, such as that of their shape. Of course, due to their limited effect to the surface potential, the resistivity values of the anomalies may not be very good.

We found that $\gamma_{11 \mathrm{n}}$ and $\gamma \mathrm{m}_{11 \mathrm{n}}$ configurations may be more productive even in the investigation of bodies that have larger effect to the potential distribution.

Finally, Fig. 11 demonstrates a realistic example (inversion parameters are in Table 1): a hole in the liner on the bottom of a waste deposit. Based on the former results, we did not find any important reason to show the $\gamma_{111}$ result in this case. The resistivity of the liner was supposed to be $10000 \Omega \mathrm{m}$, whereas that of the background was supposed to be $100 \Omega \mathrm{m}$. The liner on the bottom of the waste deposit used to be a kind of plastic that has a very high resistivity value. The hole was supposed-for simplicity reasons-to have the same resistivity such as that of the "waste" itself and the rock below the waste deposit. It is a simple model for the given situation, but it is able to handle the main point of the problem, i.e., the detectability of the hole and its positioning.

In this case, the fundamental question is whether there is a hole in the liner or none. Regarding this question, all configurations with the exception of the $\mathrm{W}-\alpha$ proved to be satisfactory because all of them presented a conductive anomaly close to the expected position that refers to the existence of a hole in the resistive liner. Regarding the second most important question, i.e., the horizontal position of the hole, in the case of the $\gamma_{\mathrm{m} 11 \mathrm{n}}$ configurations $(n=5-7)$, the hole is horizontally in the middle of the conductive anomaly, but its horizontal extension is much larger than that of the body. The Stummer configuration indicates a discontinuity in the resistive layer, but it is strongly mispositioned and much wider than the hole, i.e., the Stummer configuration was not able to localize it precisely. In contrast, the $\gamma_{\mathrm{m} 11 \mathrm{n}}$ configurations $(n=2-4)$, particularly the $\gamma_{\mathrm{m} 112}$ and $\gamma_{\mathrm{m} 113}$ ones, produced narrow anomalies at the right location. These configurations seem therefore to be convenient to detect a hole and to localize it to fulfill the most principal tasks.

Although it is not important from the point of view of the given problem, we note that the segment below the liner is resistive in the inverted section due to the fact that the current is not able to penetrate below the resistive liner. Regarding it differently, the whole bottom part of the section below the liner would be most likely below the depth of investigation (DOI) level introduced by Oldenburg and Li (1999) because the DOI level used to be closer to the surface where there are bodies with large resistivity in contrast to the average values. It refers in turn to the fact that, below this level, surface data are insensitive to the value of the physical property of the Earth.

\section{CONCLUSIONS}

A new configuration type, i.e., the $\gamma_{11 n}$ configuration, is introduced, which has not been investigated yet. Our numerous former studies let us assume that such so-called quasinull configurations can be very useful complements to the traditional configurations.

Numerical investigations showed that, although models that have a large impact to the surface potential were presented better by the traditional configurations, the quality of their image decreases faster with a decreasing model impact than that of the $\gamma_{11 \mathrm{n}}$ configurations. For small-impact models, the application of the $\gamma_{11 \mathrm{n}}$ configurations is worthwhile, at least together with a traditional configuration. It was shown that the quality of the image of $\gamma(\mathrm{m})_{11 \mathrm{n}}$ configurations can even be further improved by the MOST procedure, resulting in a good image even for small-effect models. If the application of the MOST procedure does not help avoid the uncertainties, then a combination of the geoelectric results with results of other geophysical investigations, or direct investigation of the possible inhomogeneity, is recommended.

Most of the $\gamma_{11 n}$ configurations proved to be definitely better than those of the traditional configurations in horizontal resolution investigations (especially with larger $n$ values.) The mirrored versions of the $\gamma_{11 \mathrm{n}}$ configurations (the $\gamma \mathrm{m}_{11 \mathrm{n}}$ configurations) were even better than the original configurations.

Although, in the vertical resolution studies, the separation of the anomalies directly below each other proved to be impossible for all studied configurations, certain $\gamma(\mathrm{m})_{11 \mathrm{n}}$ configurations, particularly, the $\gamma \mathrm{m}_{113}$ configuration, proved to be good in detection of the deeper bodies.

For certain models $\gamma \mathrm{m}_{11 \mathrm{n}}$, configurations may be better even in the case of large-impact inhomogeneity, as it was illustrated. The $\gamma \mathrm{m}_{11 \mathrm{n}}$ configurations proved to be better than even the Stummer configuration also in detection and positioning of a hole in the liner in a realistic field example. 
Summarizing the numerical results, it can be stated that the $\gamma(\mathrm{m})_{11 \mathrm{n}}$ configurations are more sensible to small-impact models than the traditional configurations, including the optimized Stummer configuration, giving a better image about them. They also proved to have better horizontal resolution. In the case of model bodies, below each other, many of them were able to indicate the existence of the lower body and even its vertical position in contrary to the traditional configurations. It is in accordance with the larger DD values of these configurations, which were calculated by Szalai et al. (2014). These statements are right, in spite of the smaller data coverage of these configurations, which could however be increased by the simultaneous use of different $\gamma(\mathrm{m})_{11 \mathrm{n}}$ configurations. By applying the MOST procedure by combining the images of several $\gamma(\mathrm{m})_{11 \mathrm{n}}$ configurations, the results could even be further improved.

In the present paper we mostly concentrated on models that seem to be most promising for the $\gamma_{11 \mathrm{n}}$ and $\gamma \mathrm{m}_{11 \mathrm{n}}$ configurations, according to our theoretical considerations. Based on these investigations, we propose that problems such as detection and characterization of tunnels, caves, cables, tubes, abandoned riverbeds, and lack of continuity in clay layers could be effectively solved by these configurations. Their use is also recommended in problems where false alarms are less important than high resolution, e.g., in dam investigations or waste deposit monitoring. They can be useful in any problems where small changes are expected with time, e.g., in any monitoring problems. Due to the reduced effect of the inhomogeneity below conductive or resistive layers, the $\gamma(\mathrm{m})_{11 \mathrm{n}}$ configurations should be effective in such problems.

They can be especially productive in comparison to other configurations in areas where the space available for measurements is limited.

The time required for measurements with the $\gamma(\mathrm{m})_{11 \mathrm{n}}$ configuration is moreover less than that of the traditional configurations because disregarding from the $\gamma \mathrm{m}_{112}$ configuration they contain less data points than the traditional configurations. The combined application of different $\gamma(\mathrm{m})_{11 \mathrm{n}}$ configurations is rather economic, and it can highly improve the efficiency of the measurements. A combination with traditional array results or with results of other geophysical measurements can also make it very straightforward to get the best possible interpretation.

We think, based on the presented investigations, that the $\gamma(\mathrm{m})_{11 \mathrm{n}}$ configurations might give significant contribution to the geoelectric method. Their further study is therefore highly recommended.

\section{ACKNOWLEDGEMENTS}

S. Szalai, one of the authors of this paper is a grantee of the Bolyai János Scholarship. This study was supported by the TAMOP-4.2.2.C-11/1/KONV-2012-0015 (Earthsystem) project sponsored by the EU and European Social Foundation. We are very grateful to Associate Editor Oliver Ritter and to the anonymous reviewers for enhancing the quality of this paper.

\section{REFERENCES}

Alpin L.M., Berdichevskii M.N., Vedrintsev G.A. and Zagarmistr A.M. 1966. Dipole Methods for Measuring Earth Conductivity, pp. 302. Consultants Bureau, New York.

Butler D.K. 2005. Near-Surface Geophysics, pp. 355. SEG, Tulsa, Oklahoma, USA.

Clark A.J. 1990. Seeing Beneath the Soil, pp. 176. B.T. Batshford Ltd., London.

EarthImager 2006. 2D Resistivity and IP Inversion Software Instruction Manual, pp. 137. Advanced Geosciences, Inc. Austin, Texas, USA.

Edwards L.S. 1977. A modified pseudosection for resistivity and induced-polarization. Geophysics 42, 1020-1036.

Falco P., Negro F., Szalai S. and Milnes E. 2013. Fracture characterisation using geoelectric null-configurations. Journal of Applied Geophysics 93, 33-42.

Furman A., Ferré T.P.A. and Warrick A.W. 2003. A sensitivity analysis of electrical resistance- tomography array types using analytical element modeling. Vadose Zone Journal 2, 416-423.

Furman A., Ferré T.P.A. and Warrick A.W. 2004. Optimization of ERT surveys for monitoring transient hydrological events using perturbation sensitivity and genetic algorithms. Vadose Zone Journal 3, pp. 1230-1239.

Kirsch R. 2006. Groundwater Geophysics, pp. 493. Springer.

Knödel K., Krummel H. and Lange G. 2005. Band 3. Handbuch zur Erkundung des Untergrundes von Deponien und Altlasten, 2nd edn, pp. 128. Springer.

Leontarakis K. and Apostolopoulos G.V. 2012. Laboratory study of the cross-hole resistivity tomography: the model stacking (MOST) technique. Journal of Applied Geophysics 80, 67-82.

Leontarakis K. and Apostolopoulos G.V. 2013. Model stacking (MOST) technique applied in cross-hole ERT field data for the detection of Thessaloniki ancient walls' depth.Journal of Applied Geophysics 93, 101-113.

Metwaly M., El-Qady G., Matsushima J., Szalai S., Al-Arifi N.S.N. and Taha A. 2008. Contribution of 3-D electrical resistivity tomography for landmines detection. Nonlinear Processes in Geophysics 15, 977-986.

Oldenburg D.W. and Li Y. 1999. Estimating depth of investigation in dc resistivity and IP surveys. Geophysics 64(2), 403416.

Roy A. and Apparao A. 1971. Depth of investigation in direct current methods. Geophysics 36, 943-959. 
Stummer P., Maurer H. and Green A.G. 2004. Experimental design: electrical resistivity data sets that provide optimum subsurface information. Geophysics 69(1), 120-139.

Szalai S., Koppán A., Szokoli K. and Szarka L. 2013a. Geoelectric imaging properties of traditional arrays and of the optimized Stummer configuration. Near-Surface Geophysics 11, 51-62.

Szalai S., Kósa I., Nagy T. and Szarka L. 2009a. Effectivity enhancement of azimuthal geoelectric measurements in determination of multiple directions of subsurface fissures, on basis of analogue modelling experiments. 15th European Meeting of Environmental and Engineering Geophysics, Proceedings \& Exhibitors' Catalogue Near Surface 2009, P25.

Szalai S., Lemperger I., Metwaly M., Kis Á., Wesztergom V., Szokoli K. and Novák A. 2014. Multiplication of the depth of detectability using $\gamma_{11 \mathrm{n}}$ arrays. Journal of Applied Geophysics 107, 195206.

Szalai S., Novák A. and Szarka L. 2009b. Depth of investigation and vertical resolution of surface geoelectric arrays. Journal of Engineering and Environmental Geophysics 14, 15-23.

Szalai S., Novák A. and Szarka L. 2011. Which geoelectric array sees the deepest in a noisy environment? Depth of detectability values of multielectrode systems for various two-dimensional models. Physics and Chemistry of the Earth 36, 1398-1404.

Szalai S., Szarka L., Marquis G., Sailhac P., Kaikkonen P. and Lahti I. 2004. Colinear null arrays in Geoelectrics. Proceedings of the 17th
S.3-P.3 IAGA WG 1.2 on Electromagnetic Induction in the Earth Workshop, Hyderabad, India.

Szalai S., Szarka L., Prácser E., Bosch F., Müller I. and Turberg P. 2002. Geoelectric mapping of near-surface karstic fractures by using null-arrays. Geophysics 67, 1769-1778.

Szalai S. and Szarka L. 2008a. On the classification of surface geoelectric arrays. Geophysical Prospecting 56, 159-175.

Szalai S. and Szarka L. 2008b. Parameter sensitivity maps of surface geoelectric arrays, I. linear arrays. Acta Geodaetica et Geophysica Hungarica 43(4), 419-437.

Szalai S. and Szarka L. 2008c. Parameter sensitivity maps of surface geoelectric arrays, II. Nonlinear and focussed arrays. Acta Geodaetica et Geophysica Hungarica 43(4), 439-447.

Van Nostrand R.G. and Cook K.L. 1966. Interpretation of resistivity data. In: Geological Survey Professional Paper 499. U.S. Government Printing Office, Washington DC.

Ward S.H. 1990. Geotechnical and Environmental Geophysics, Vol. I. Review and Tutorial. SEG, Tulsa, Oklahoma, USA.

Wilkinson P.B., Meldrum P.I., Chambers J.E., Kuras O. and Ogilvy R.D. 2006. Improved strategies for the automatic selection of optimised sets of electrical resistivity tomography measurement configurations. International Journal of Geophysics, 1119 1126.

Zhdanov M.S. and Keller G.V. 1993. The Geoelectrical Methods in Geophysical Exploration, pp. 973. Elsevier. 